\title{
PARAMETER MEASUREMENT OF BIAXIAL BRAIDED COMPOSITE PREFORM BASED ON PHASE CONGRUENCY
}

\author{
Zhitao Xiao ${ }^{1,2}$, Lei $\mathrm{Pei}^{2}$, Fang Zhang ${ }^{1,2^{\star}}$, Ying Sun ${ }^{3}$, Lei Geng ${ }^{1,2}$, Jun $\mathrm{Wu}^{1,2}$, Jun Tong ${ }^{4}$ \\ ${ }^{1}$ Tianjin Key Laboratory of Optoelectronic Detection Technology and System, Tianjin, P.R. China \\ ${ }^{2}$ School of Electronics and Information Engineering, Tianjin Polytechnic University, Tianjin, P.R. China \\ ${ }^{3}$ Institute of Textile Composites, Tianjin Polytechnic University, Tianjin, P.R. China \\ ${ }^{4}$ School of Electrical, Computer and Telecommunications Engineering, University of Wollongong, Wollongong, Australia \\ *Email: hhzhangfang@126.com
}

\begin{abstract}
:
In this paper, a new method based on phase congruency is proposed to measure pitch lengths and surface braiding angles of two-dimensional biaxial braided composite preforms. Lab space transform and BM3D (block-matching and $3 D$ filter) are used first to preprocess the original acquired images. A corner detection algorithm based on phase congruency is then proposed to detect the corners of the preprocessed images. Pitch lengths and surface braiding angles are finally measured based on the detected corner maps. Experimental results show that our method achieves the automatic measurement of pitch lengths and the surface braiding angles of biaxial braided composite preforms with high accuracy.
\end{abstract}

\section{Keywords:}

$2 D$ biaxial braided composite preform, parameter measurement, surface braiding angle, pitch length, phase congruency

\section{Introduction}

Compared with conventional unidirectional laminated composites, textile composites have been widely applied in the aerospace, sports, automobile, and marine industries due to their advanced performance, including higher wholeness, less fabrication costs, greater shear strengths, and better impact resistance characteristics. ${ }^{[1]}$ Composite structures are gradually replacing metal components in the automotive and aeronautic industry for their significant potential in weight reduction and design flexibility. ${ }^{[2]}$

Braiding has been used since 1800s for producing textile fabrics. ${ }^{\left[{ }^{3]}\right.}$ In the braiding process, the strands are deposited onto a centrally placed, transverse moving mandrel via strand carriers that rotate around the mandrel. ${ }^{[4]}$ Constant or varying axisymmetric cross-section mandrels can be used..$^{[5]}$ Braided structures can be classified as two-dimensional (2D) braids produced on the conventional maypole braiders and three-dimensional (3D) through-thickness braids produced on specialized machinery. ${ }^{[6]}$ Two-dimensional braids can be classified into diamond braid (1/1), regular braid (2/2), and Hercules braid (3/3), according to the interlacement of yarns (see Figure 1) ${ }^{[7]}$ Diamond braids have an alternation of one tow passing above and then below the other tows, regular braids pass above two and below two tows in a repeat, and Hercules braids have a structure of three tows up and three tows down. ${ }^{[7]}$

The pitch length $h$ and surface braiding angle $\theta$ are two important parameters of braided composites, which can be used for predicting the mechanical properties of braided composites and affect the micro-structures of braided composites as well. ${ }^{[8]}$
The pitch length $h$ is defined as the length formed in a complete machine cycle along the braiding direction. ${ }^{[9]}$ The surface braiding angle $\theta$ is the angle between the braided yarns and the braiding direction on the fabric surface..$^{[10,11]}$ (See Figure 2).

References [11-13] studied the automatic measurement of pitch lengths and surface braiding angles for $3 D$ braided composite preforms. However, there is limited research on the automatic measurement for 2D braided composite preforms. Manual measurement of the pitch length and surface braiding angle, which is still prominent in industry, is time-consuming, manpower-costing, and prone to errors. Therefore, it is significant to achieve the automatic measurement of the two parameters. In this paper, a method based on phase congruency is proposed to tackle this problem for biaxial, regular braided composite preforms.

\section{Experimental}

\subsection{Materials}

The braided tubes considered include 2D biaxial regular braided carbon, glass and aramid fiber composite preforms. The average diameters of the carbon, glass and aramid fiber filaments are, respectively, $5.70 \mu \mathrm{m}, 7.91 \mu \mathrm{m}$ and $11.02 \mu \mathrm{m}$, tested by the College of Textiles of Tianjin Polytechnic University using the fiber fineness analyzer manufactured by Laizhou Electron Instrument CO., LTD. (Taiyuan, China). As shown in Figure 3, the parts of fibers are cut as the sample and placed on the slide glass covered by a cover glass. The sample is then placed under the microscope of the fiber fineness analyzer. The 


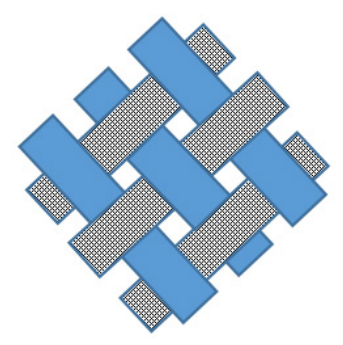

(a) Diamond braid (1/1)

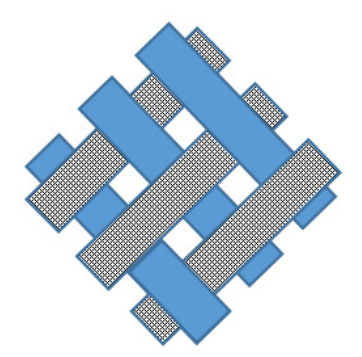

(b) Regular braid (2/2)

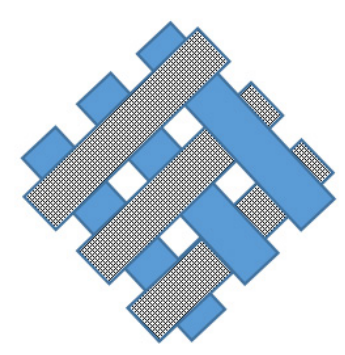

(c) Hercules braid (3/3)

Figure 1. Structures of $2 \mathrm{D}$ braids

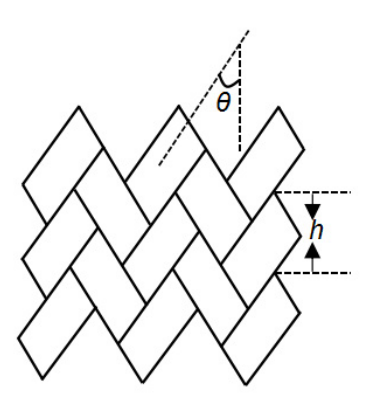

(a) Ideal scenario

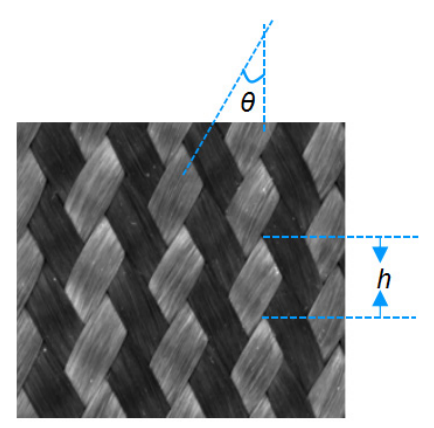

(b) Practical scenario

Figure 2. Pitch length and surface braiding angle

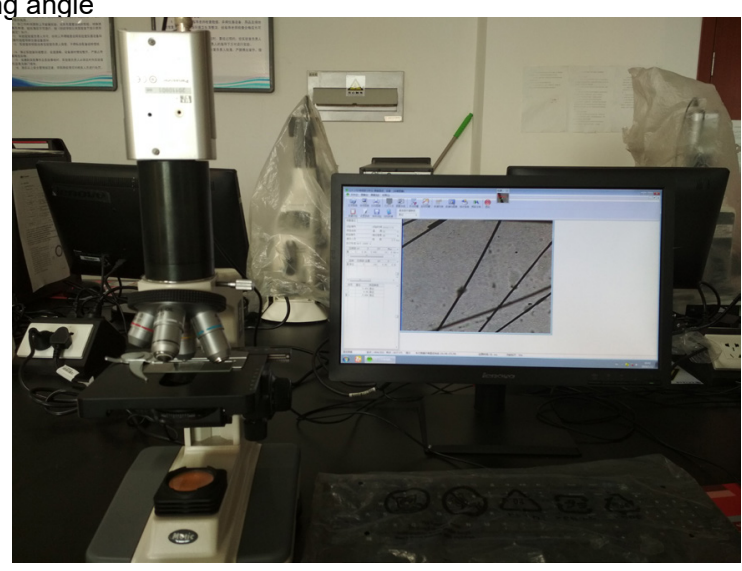

Figure 3. Fiber fineness analyzer

magnification and focus of the microscope are tuned to obtain a clear fiber image on the computer screen. Finally, a point is clicked on each side of the fiber image along the diameters direction, and the diameter is computed by the software. This step is repeated to get the average fiber diameter.

Since the braided tubes tend to deform, they are pasted on the horizontal plates by the double-sided tapes, as illustrated in Figure 4.

\subsection{Image acquisition}

The original images are acquired by the image acquisition system illustrated in Figure 5, which consists of a dome light source (DLS), a circular polarizing filter (CPF), a CCD camera, and a microcaliper. While acquiring the images, the CCD camera is vertically installed above the fabric, the CPF is fixed in front of the camera lens, and the microcaliper is put on one side of the fabric, keeping the surfaces of the microcaliper and the fabric on the same horizontal plane. The DLS is then put in front of the fabric, making the camera's optical center go through the central axis of the light hole of DLS and the center of the fabric surface as well. Finally, the CPF is rotated, the camera exposure value is adjusted, and a clear image is selected and saved. The model and parameters of the devices used are shown in Table 1.

From Figure 6, we can see that using the CPF and DLS can improve the contrast and lightness of the images acquired.

\subsection{Image preprocessing}

The acquired images are preprocessed by the Lab transform ${ }^{[14]}$ and a block-matching and $3 D$ filter $(B M 3 D)^{[15]}$ to enhance the contrast and filter noise. The CIE-Lab color space was introduced by the Commission Internationale de L'Eclairage (CIE) in 1976. It has three axes: $L$, $a$ and $b$, where $L$ axis represents light while $a$ and $b$ axes are color channels. $L, a$ and 


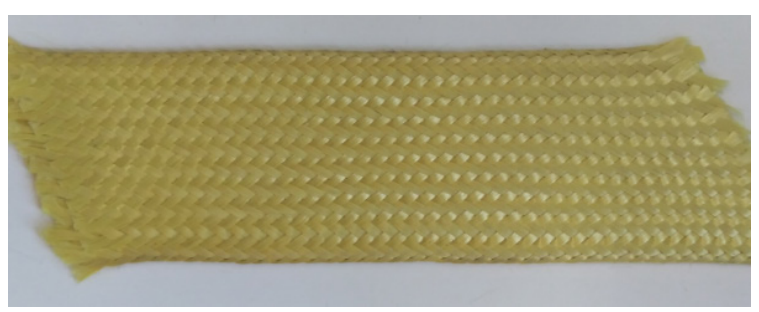

Figure 4. Aramid fabric sample
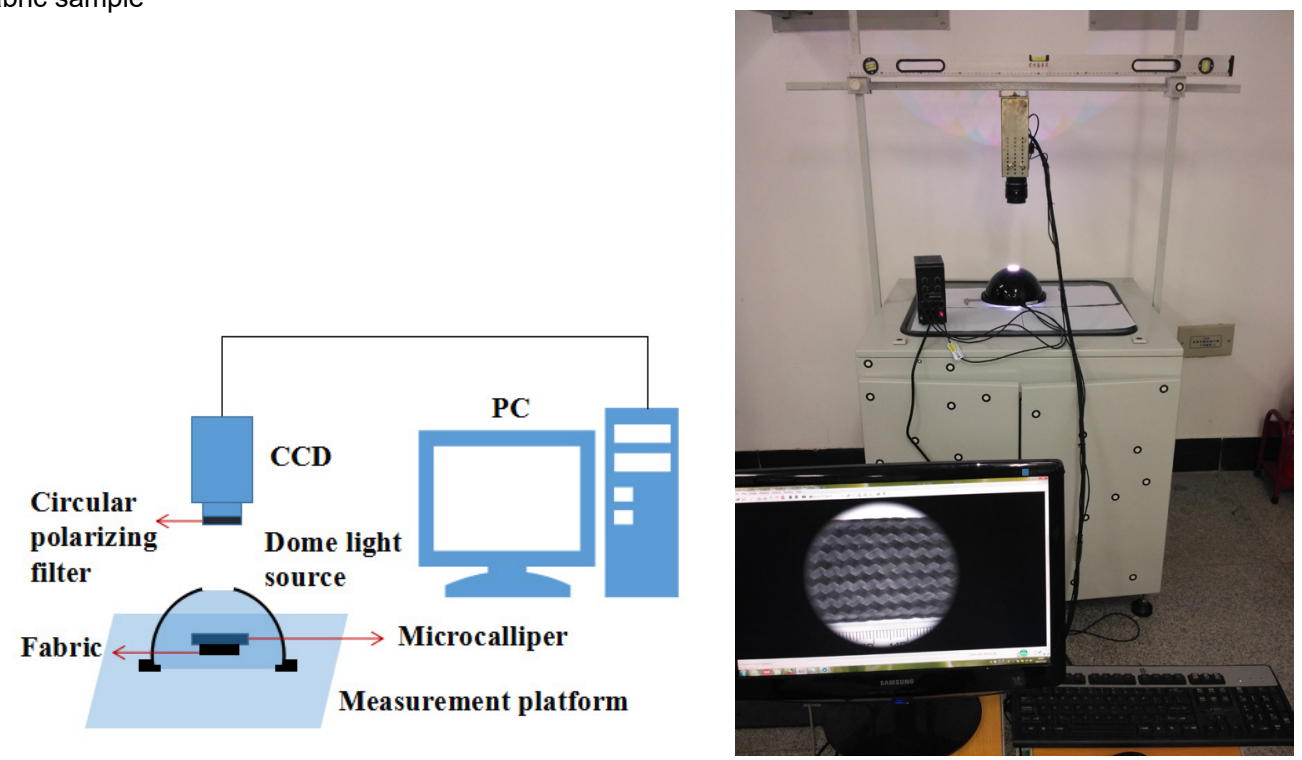

(a) The system diagram

(b) The actual system

Figure 5. Image acquisition system

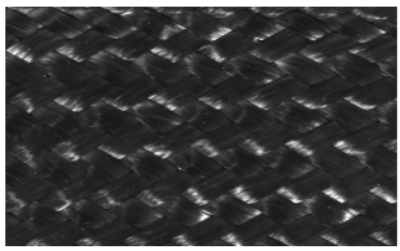

(a) Without CPF and without DLS

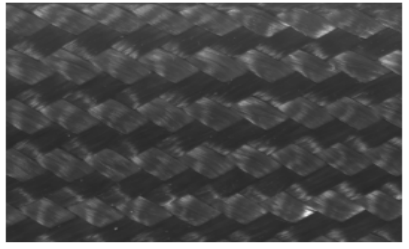

(b) With CPF and without DLS

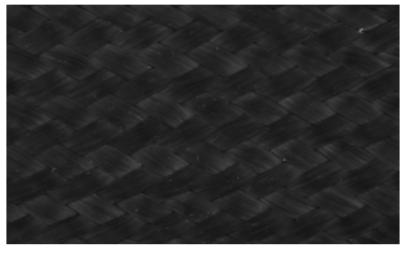

(c) Without CPF and with DLS

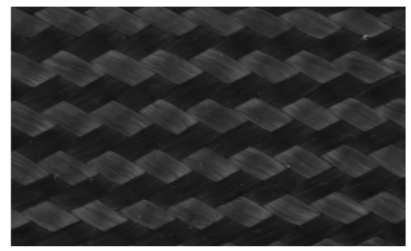

(d) With CPF and DLS

Figure 6. Images acquired using different image acquisition schemes

Table 1. Devices and relevant parameters

\begin{tabular}{|c|c|}
\hline Devices & Parameters \\
\hline Dome light source & OPT-RID180-RGB \\
\hline CCD camera & Vieworks VA-8MC-M16AO \\
\hline Camera lens & Nikkor 28mm f/2.8D \\
\hline Circular polarizing filter & NiSi CPL 52mm \\
\hline PC & HP Pro 3335MT; CPU: AMD Athlon; (tm) X2 B28 \\
\hline
\end{tabular}

$b$ were converted from $R, G$ and $B$ space, using Eqs. (1)-(5) as follows. ${ }^{[14]}$

$$
\left(\begin{array}{l}
X \\
Y \\
Z
\end{array}\right)=\left[\begin{array}{lll}
0.412453 & 0.357580 & 0.180423 \\
0.212671 & 0.715160 & 0.072169 \\
0.019334 & 0.119193 & 0.950227
\end{array}\right]\left(\begin{array}{l}
R \\
G \\
B
\end{array}\right)
$$

$$
\begin{aligned}
& L=116\left[f\left(\frac{Y}{Y_{w}}\right)\right]-16 \\
& a=500\left[f\left(\frac{X}{X_{w}}\right)-f\left(\frac{Y}{Y_{w}}\right)\right] \\
& b=200\left[f\left(\frac{Y^{w}}{Y_{w}}\right)-f\left(\frac{Z^{\prime}}{Z_{w}}\right)\right]
\end{aligned}
$$




$$
f(t)= \begin{cases}t^{\frac{1}{3}} & \text { if } t>\left(\frac{6}{29}\right)^{3} \\ \frac{1}{3}\left(\frac{29}{6}\right)^{2} t+\frac{4}{29} & \text { if } t \leq\left(\frac{6}{29}\right)^{3}\end{cases}
$$

where $X w=0.9504, Y w=1.0000$ and $Z w=1.0887$ are the tristimulus of $\mathrm{CIE} X, Y$ and $Z$ values with reference to the "white spot". If the acquired image is a gray scale image, it is first turned into an equivalent $R G B$ image (with the same values in the three channels) and then converted to the $L$, $a$ and $b$ space. ${ }^{[14]}$ We extract the $L$ channel image to achieve image enhancement. BM3D, which filters noise in the 3D transform domain by combining sliding-window transform processing with block-matching, ${ }^{[15]}$ is then used to filter noise of the Lab transformed image. As shown in Figure 7, the image $F_{B M 3 D}$ preprocessed by Lab transform and BM3D has higher contrast and weaker texture.

\subsection{Corner detection based on phase congruency}

After preprocessing, image $F_{B M 3 D}$ is then processed for detecting corners based on phase congruency. The detailed steps are described as follows.

Step 1: Calculate the minimum moment of the phase congruency covariance $([16,17])$ of $F_{B M 3 D}$, getting image $F_{P C m}$ (Figures $8(d)$ and $9(d)$ ).

Step 2: Binarize $F_{P C m}$ using a threshold $T=0.25$, obtaining image $F_{T}$ (Figures $8(\mathrm{e})$ and $\left.9(\mathrm{e})\right)$ :

$$
F_{T}(i, j)= \begin{cases}255, & F_{P C m}(i, j) \geq T \\ 0, & \text { otherwise }\end{cases}
$$

Step 3: Remove false corners based on the principle that the gray value of the true corner is smaller than other corners' in certain neighborhoods: first, scan $F_{T}$ with $11 \times 11$ neighborhoods centered on the corners detected in $F_{T}$; then in the neighborhoods, compare all corners' gray values in $F_{L}$ and reserve only corners with the minimum gray values. Repeat this procedure using $F_{P C m}$ in $7 \times 7$ neighborhoods, getting corner map $F_{\text {ocor }}($ Figures $8(\mathrm{~g})$ and $9(\mathrm{~g})$ ).

Step 4: Further remove the false corners based on image autocorrelation, ${ }^{18]}$ obtaining the image $F_{\text {ocor }}$. In this step, an approximate value $T h$ of pitch length will be used, based on which the image autocorrelation ${ }^{[18]}$ reflects the cyclic changes of the fabric texture, and the periods are approximately equal to the pitch length in pixels. Thus, Th can be calculated as follows: Firstly, calculate each row's autocorrelation map $F_{A C i}$ of $F_{L}(i=1$, $2,3, \ldots, m ; m$ is the number of rows of $F_{L}$ ); Secondly, calculate the average peak-to-peak value $T h_{i}$ of the three peaks located in the middle of $F_{A C i}$, and then choose the maximum value $T h_{\max }$ $=\max \left\{T h_{i}\right\}$ as a reference value; Thirdly, in $F_{A C{ }^{\prime}}$ if there are three peaks in the interval $\left[P_{m i}-T h_{m a x}, P_{m i}+T h_{\text {max }}\right]\left(P_{m i}\right.$ is the position of the middle peak), then save this $T h_{i}$; Finally, calculate the average value of the saved $T h_{i}$ as $T h$.

Let $A_{1}\left(x_{1}, y_{1}\right), A_{2}\left(x_{2}, y_{2}\right)$ and $A_{3}\left(x_{3}, y_{3}\right)$ be the three adjacent corners. Define $d_{12}$ and $d_{23}$ as the distance between $A_{1}$ and $A_{2}$, and, $A_{2}$ and $A_{3}$, respectively. There are two cases when removing false corners in $F_{\text {ocor }}$ :

Case 1: Remove corner $A_{1}$ if either of the following conditions is met:

- $\left|d_{23}-k^{*} T h\right|<7+3(k-1)$ and $d_{12}<T h-10(k=1,2,3, \ldots)$.

- $\left|d_{23}-k^{*} T h\right|<7+3(k-1)$ and $I^{*} T h_{\text {in }}+T h_{\text {ind10 }}+1.5+0.5(l-1)<d_{12}$ $<(l+1)$ Th- $(15+5(l-1)), k=1,2,3, \ldots ; l=1,2,3, \ldots ;$; where $T h_{\text {in }}$ is the integer part of $T h$ and $T h_{i n d 10}$ is the integer part of $(1 / 10) T h$.

Case 2: Remove corner $A_{2}$ if either of the following conditions is met:

- $\quad d_{12}<(1 / 3) T h$ and $(1 / 3) T h<d_{23}<T h-10$.

- $\quad d_{23}<(1 / 3)$ Th and $(1 / 3)$ Th $<d_{12}<$ Th-10.

Case 3: Remove corner $A_{3}$ if either of the following conditions is met:

- $\quad\left|d_{12}-k^{\star} T h\right|<7+3(k-1)$ and $d_{23}<T h-10(k=1,2,3, \ldots)$.

- $\left|d_{12}-k^{*} T h\right|<7+3(k-1)$ and $I^{*} T h_{\text {in }}+T h_{\text {ind } 10}+1.5+0.5(l-1)<d_{23}<$ $(I+1)$ Th- $(15+5(I-1)), k=1,2,3, \ldots ; I=1,2,3, \ldots$.

Step 5: Fill missing corners for $F_{\text {ocor }}$ :

Case 1: Missing corners are assumed between two known corners.

Let $d_{12}$ be the distance between two known corners $A_{1}\left(x_{1}, y_{1}\right)$ and $A_{2}\left(x_{2}, y_{2}\right)$. If $k^{*} T h+(2 / 3) T h<d_{12}<(k+1) T h+(1 / 3) T h$, then assume $k$ missing corners $(k=1,2,3,4, \ldots)$ between $A_{1}$ and $A_{2}$. The coordinates $\left(x_{m}, y_{m}\right)$ of one missing corners are calculated by

$$
y_{m}=\left(y_{1}+y_{2}\right) / 2
$$
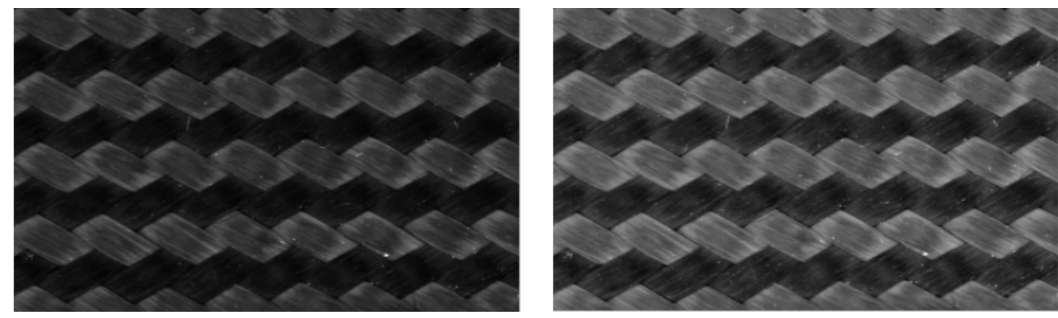

(a) Original image $F_{\text {ori }}$

(b) L channel image $F_{L}$

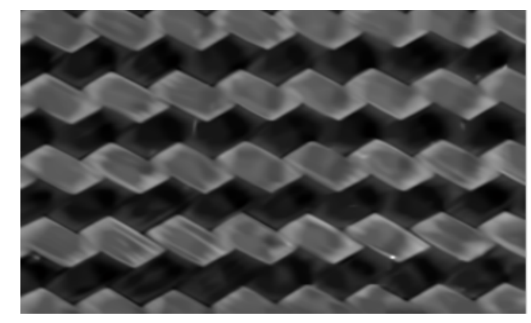

(c) BM3D filtered image $F_{\text {BM3D }}$

Figure 7. Image preprocessing 


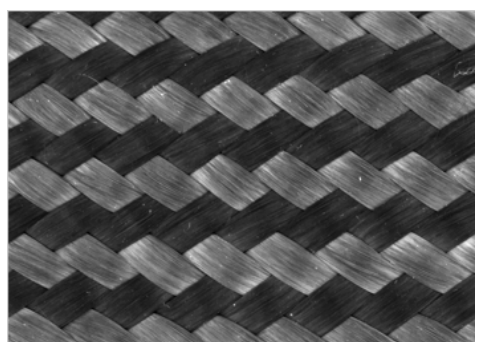

(a) $\mathrm{F}_{\text {or }}$

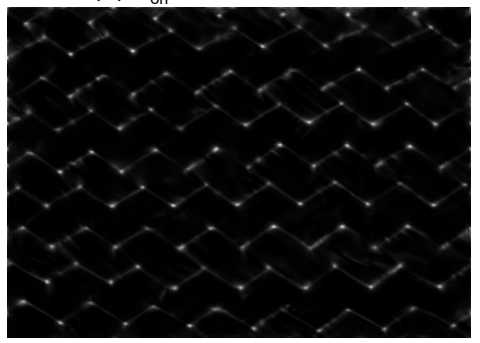

(d) $\mathrm{F}_{\mathrm{PCm}}$

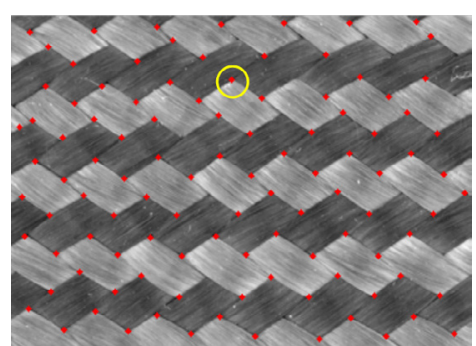

(g) $\mathrm{F}_{\text {ocor }}$

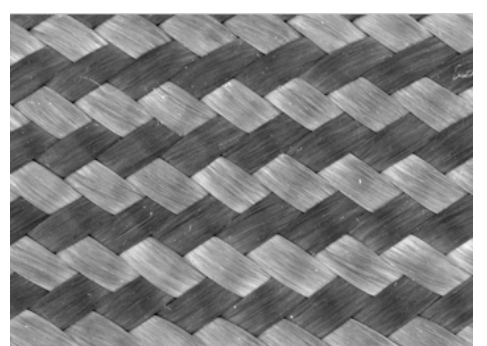

(b) $\mathrm{F}_{\mathrm{L}}$

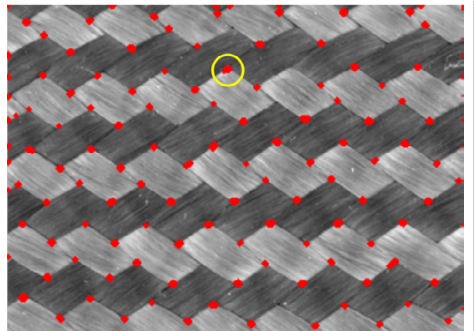

(e) $F_{T}$

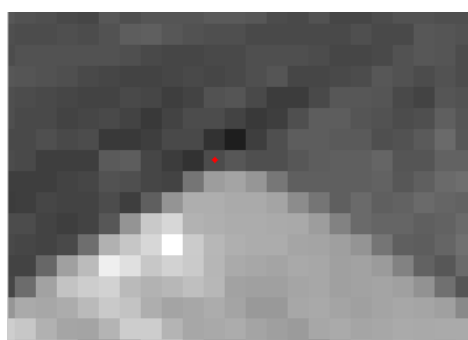

(h) Part of $F_{\text {ocor }}$ marked by yellow circle in $(\mathrm{g})$

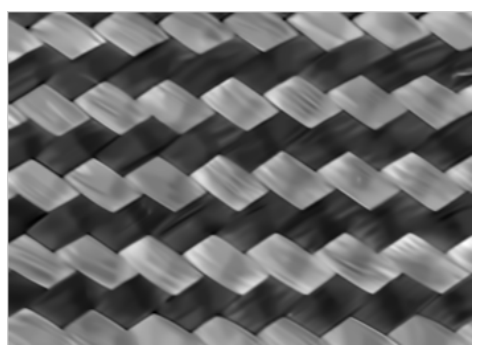

(c) $F_{B M 3 D}$

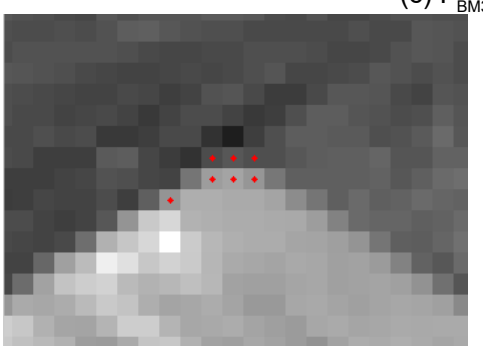

(f) Part of $F_{T}$ marked by yellow circle in (e)

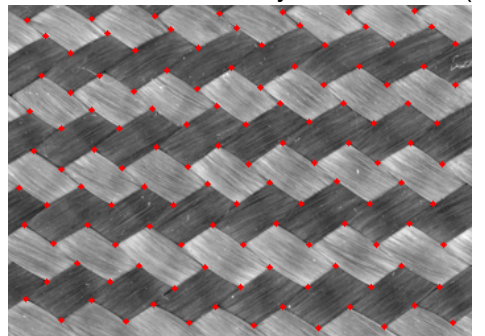

(i) $\mathrm{F}_{\text {fcor }}$

Figure 8. Corner detection process of carbon fiber fabric

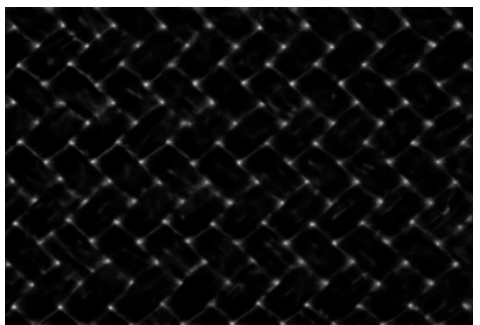

(d) $F_{P C}$

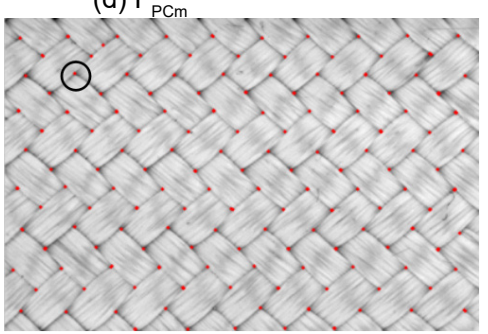

(g) $\mathrm{F}_{\text {ocor }}$

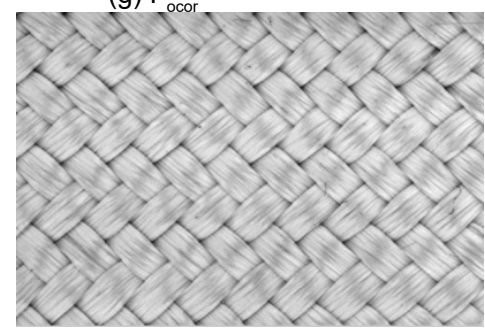

(j) $F_{\text {ori }}$

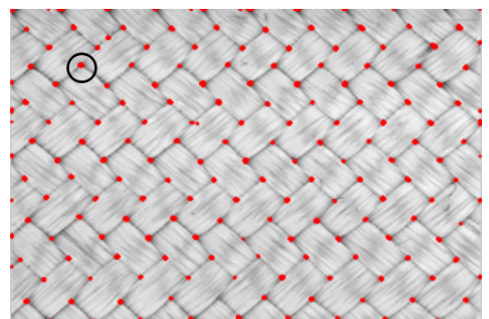

(e) $F_{T}$

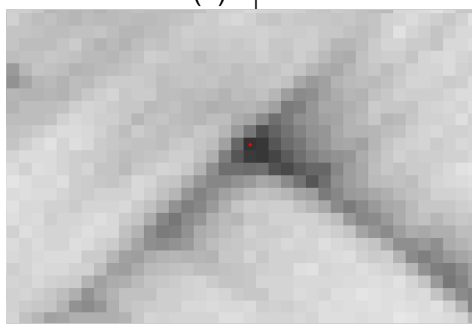

(h) Part of $\mathrm{F}_{\text {ocor }}$ marked by yellow circle in $(\mathrm{g})$

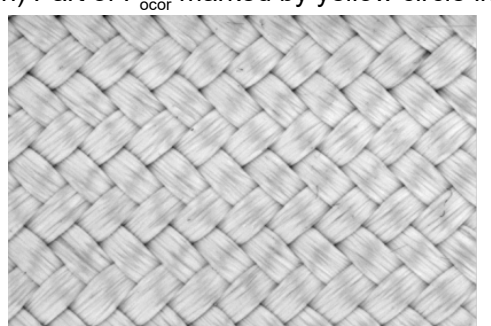

(k) $F_{L}$

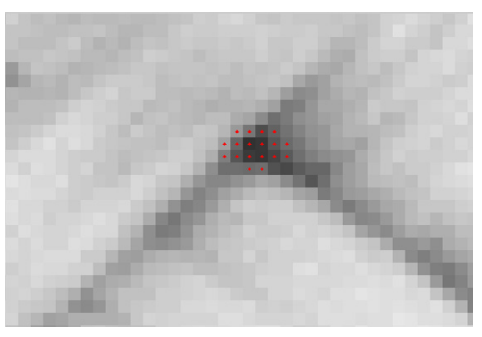

(f) Part of $F_{T}$ marked by yellow circle in (e)

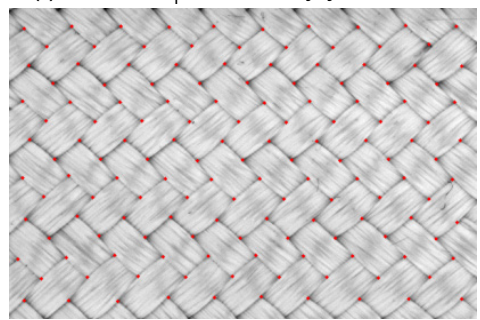

(i) $\mathrm{F}_{\text {fcor }}$

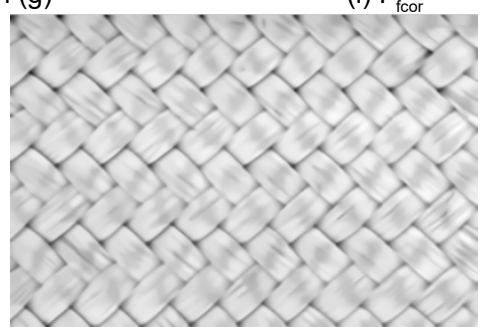

(I) $F_{B M 3 D}$

Figure 9. Corner detection process of glass fiber fabric 


$$
x_{m}= \begin{cases}\left(y_{m}-b\right) / a, & x_{1} \neq x_{2} \\ x_{1}, & x_{1}=x_{2}\end{cases}
$$

The coordinates $\left(x_{m 1}, y_{m 1}\right)$ and $\left(x_{m 2}, y_{m 2}\right)$ of the two missing corners are calculated by

$$
\begin{aligned}
& y_{m 1}=y_{1}+h \\
& y_{m 2}=y_{1}+2 h \\
& x_{m 1}= \begin{cases}\left(y_{m 1}-b\right) / a, & x_{1} \neq x_{2} \\
x_{1}, & x_{1}=x_{2}\end{cases} \\
& x_{m 2}= \begin{cases}\left(y_{m 2}-b\right) / a, & x_{1} \neq x_{2} \\
x_{1}, & x_{1}=x_{2}\end{cases}
\end{aligned}
$$

where $a$ and $b$ are respectively, the slope and intercept of the straight line connecting $A_{1}$ and $A_{2} h=d_{12} /(k+1)$. The cases with more missing corners can be handled similarly. Finally, adjust the positions of these detected missing corners; compare all the gray values of the image $F_{L}$ in $3 \times 3$ neighborhoods centered on each detected missing corner; and choose those with minimum gray values as new corners.

\section{Case 2: Missing corners are assumed between the leftmost} corner and left edge of the image.

Let $d_{c e l}$ be the distance between the leftmost corner and the left edge. If $k^{\star} T h+(1 / 20) T h<d_{c e l}<(k+1) T h$, then assume $k$ missing corners between the leftmost corner and the left edge $(k=1$, $2,3, \ldots)$.

Case 2.1: More than one known corner in a line.

Let $A_{l}\left(x_{l}, y_{l}\right)$ and $A_{r}\left(x_{r}, y_{r}\right)$ be the known leftmost corner and the known rightmost corner in the same line, respectively. Then the coordinates of a single missing corner $\left(x_{e l}, y_{e l}\right)$ is computed by:

$$
\begin{aligned}
& y_{e l}=y_{l}-h \\
& x_{e l}= \begin{cases}\left(y_{e l}-b_{l r}\right) / a_{l r}, & x_{l} \neq x_{r} \\
x_{l}, & x_{l}=x_{r}\end{cases}
\end{aligned}
$$

where $h=\min \{d a, T h\}, d a$ is the average distance between adjacent known corners; $a_{l r}$ and $b_{l r}$ are respectively, the slope and intercept of the line determined by corner $A_{1}$ and $A_{r}$. The coordinates of the two missing corners $\left(x_{e / 1}, y_{e / 1}\right)$ and $\left(x_{e / 2}, y_{e / 2}\right)$ are computed by

$$
\begin{aligned}
& y_{e / 1}=y_{l}-h \\
& y_{e / 2}=y_{l}-2 h \\
& x_{e / 1}= \begin{cases}\left(y_{e / 1}-b_{l r}\right) / a_{l r}, & x_{l} \neq x_{r} \\
x_{l}, & x_{l}=x_{r}\end{cases} \\
& x_{e / 2}= \begin{cases}\left(y_{e / 2}-b_{l r}\right) / a_{l r}, & x_{l} \neq x_{r} \\
x_{l}, & x_{l}=x_{r}\end{cases}
\end{aligned}
$$

Similarly, more than two missing corners can be handled.

Case 2.2: One known corner in a line

$\left(x_{e l}, y_{e l}\right),\left(x_{e / 1}, y_{e / 1}\right)$ and $\left(x_{e / 2}, y_{e / 2}\right)$ can be computed similarly with Eqs. (13)-(18), the difference is here $h=T h$.

Case 3: Missing corners are assumed between the rightmost corner and right edge of the image. The treatment is similar to Step 5 of Case 2.

Finally, adjust the filled missing corners using $F_{P C m}$ in $7 \times 7$ neighborhoods, getting corner map $F_{\text {fucor' }}$ similar to Step 5 of Case 1.

Step 6: Adjust all the corners in $3 \times 3$ neighborhoods based on image $F_{L}$, getting image $F_{\text {fcor }}$, which is similar with the Case 1 of Step 5.

Figures 8 and 9 illustrate the corner detection process of carbon and glass fiber fabric, respectively.

\subsection{Measurement of pitch lengths and surface braiding angles}

Pitch lengths and surface braiding angles are measured based on the detected corner map $F_{\text {fcor }}$. As shown in Figure 10, the pitch length $d_{\text {AiAjpl }}$ can be computed by

$$
d_{A i A j p l}=d_{A i A j} \times d_{C}
$$

where $d_{A i A j}$ is the distance between corner $A_{i}$ and $A_{j}(i=1,2,3$, $\ldots ; j=i+1)$, and $d c$ is the actual length per pixel determined from

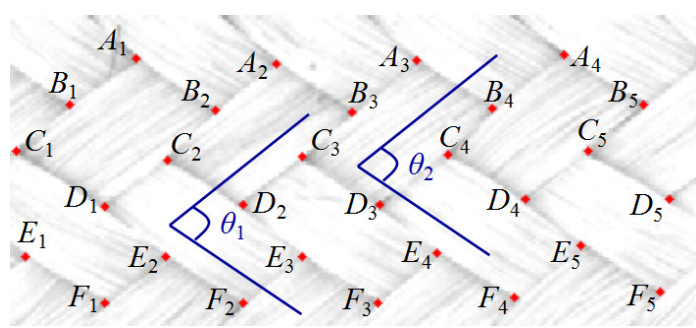

Figure 10. Labeled corners and angle of aramid fiber 
image calibration. Similarly, the pitch lengths $d_{B i B j p l}, d_{C i C j p l}$ and $d_{D i D j p l}$ can be calculated.

The surface braiding angles $(1 / 2) \angle \theta_{1}$ and $(1 / 2) \angle \theta_{2}$ can be calculated by

$$
\begin{aligned}
& (1 / 2) \angle \theta_{1}=(1 / 2) \angle A_{2} C_{2} D_{2} \\
& (1 / 2) \angle \theta_{2}=(1 / 2) \angle C_{4} D_{3} F_{4}
\end{aligned}
$$

\section{Results and discussion}

In this section, four sample images are tested using the proposed method. Figure $11(\mathrm{a})-(\mathrm{d})$ are respectively for carbon, carbon, glass and aramid-fiber fabric cropped from the original images acquired in Section 2.2. The measured angles are labeled in Figure 11(e) - (h). In Figure 11(e), for example, the measured surface braiding angle are $(1 / 2) \angle \theta_{i}(i=1,2,3, \ldots$, 18), and the measured pitch lengths are $d_{A i A j p l}=d_{c} \times d_{A i A j}(i=1$, $2, \ldots ; j=i+1)$, where $d_{A i A j}$ is the pixel distance between corners $A i$ and $A_{i j}$. Pitch lengths $d_{B i B j p l}$ in the second line and other lines are measured in the same way.

With manual measurements, $d_{A i A j}$ is measured by clicking relevant points on the image and $\theta_{i}$ is measured using the protractor. For the example in Figure 12(a), the coordinates of corner $A(109,157)$ and $B(109,204)$ are acquired by clicking

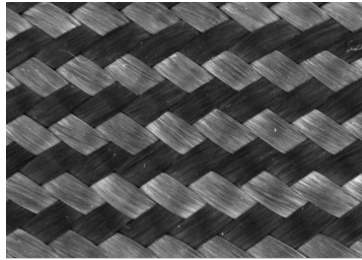

(a) Image $F_{1}$

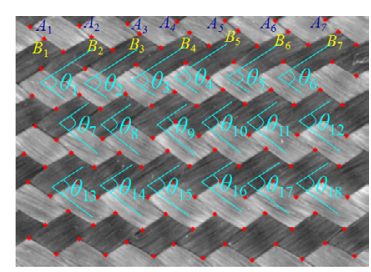

(e) Labeled angles and corners of $F_{1}$

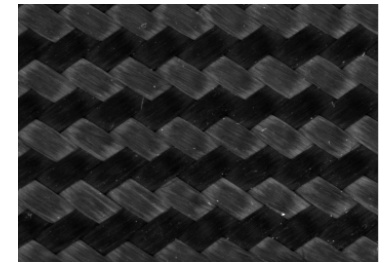

(b) Image $F_{2}$

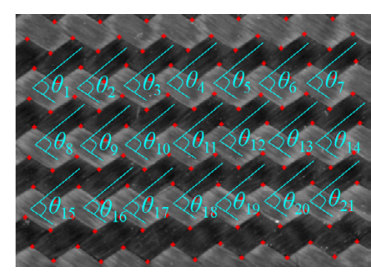

(f) Labeled angles of $F_{2}$

Figure 11. Tested images and labeled angles and corners

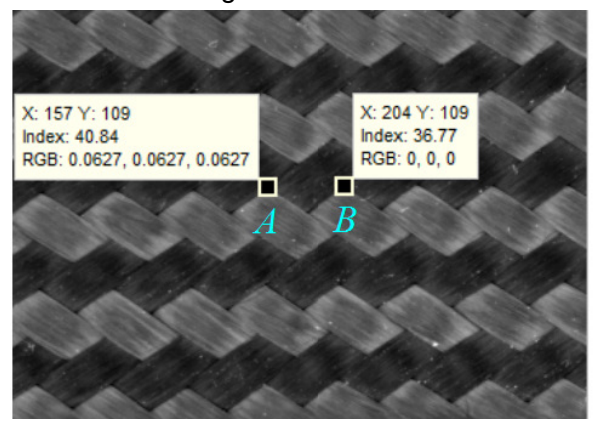

(a)

points on the image of computer screen and the pitch length is computed as $d_{A B \rho l}=d_{c} \sqrt{(109-109)^{2}+(157-204)^{2}}$. In Figure 12(b), the angle $\theta$ measured by protractor is $73.05^{\circ}$, and the surface braiding angle is $(1 / 2) \angle \theta=(1 / 2) 73.05=36.525^{\circ}$. Each pitch length and surface braiding angle are measured manually for ten times and the averages are taken as the final results.

Manual measurements of the pitch length and surface braiding angle are used to benchmark the proposed automatic measurement method due to their small standard deviations as shown in Table 2.

Figure 13 plots the errors (ordered in the ascending order) of our proposed method relative to manual measurements. Table 3 gives the average values of the pitch length and surface braiding angle measurements and the relative errors, where $d_{a h}$ and $d_{a o}$ are the average values of the pitch lengths measured by hands and by our method, respectively, $\theta_{a h}$ and $\theta_{a o}$ are the average values of the surface braiding angles measured by hands and by our method, respectively, and $e_{d a}$ and $e_{\theta a}$ are, respectively, the errors of the average pitch length and surface braiding angle relative to manual measurements.

From Figure 13 and Table 3, the following observations can be made:

- The proposed method achieves automatic measurement of pitch lengths and surface braiding angles of 2D biaxial

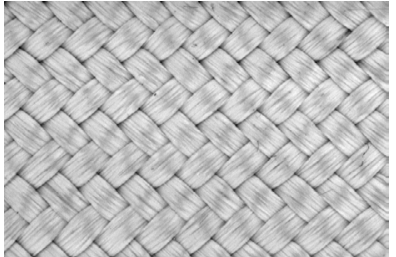

(c) Image $\mathrm{F}_{3}$

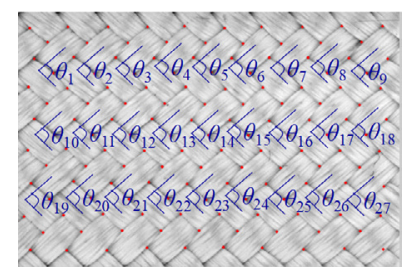

(g) Labeled angles of $\mathrm{F}_{3}$

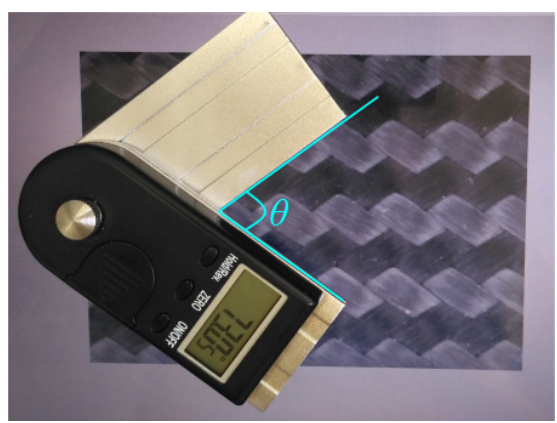

(b)

Figure 12. Manual measurement of (a) pitch length and (b) surface braiding angle 

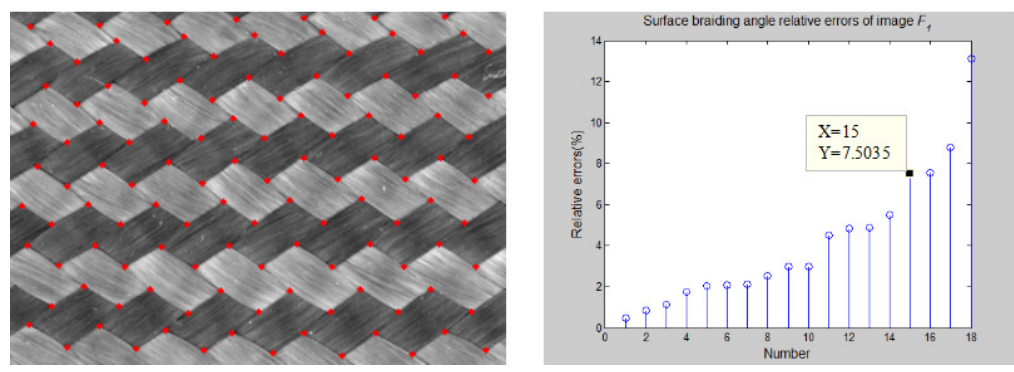

(a) Results of corner detection of image $F_{1}$

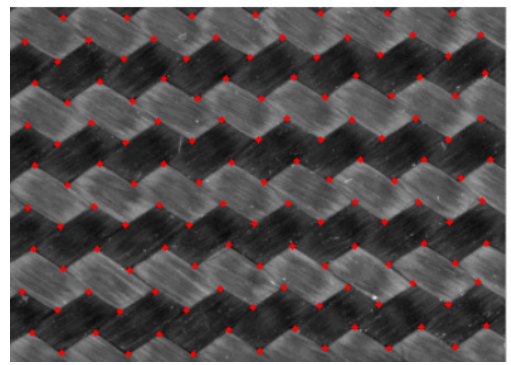

(b) Relative errors of measuring surface braiding angles of $F_{1}$

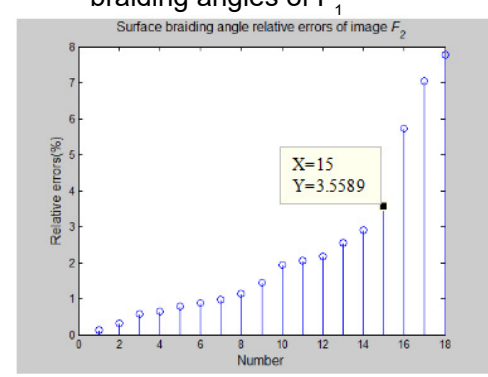

(d) Results of corner detection of image $F_{2}$

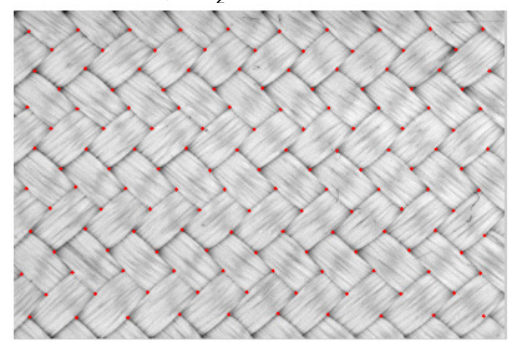

(g) Results of corner detection of image $\mathrm{F}_{3}$

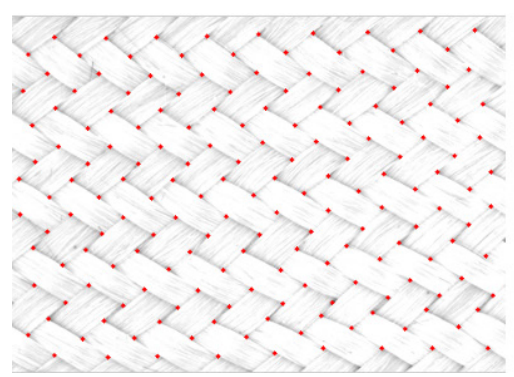

(j) Results of corner detection of image $\mathrm{F}_{4}$

(k) Relative errors of measuring surface braiding angles of $\mathrm{F}_{4}$

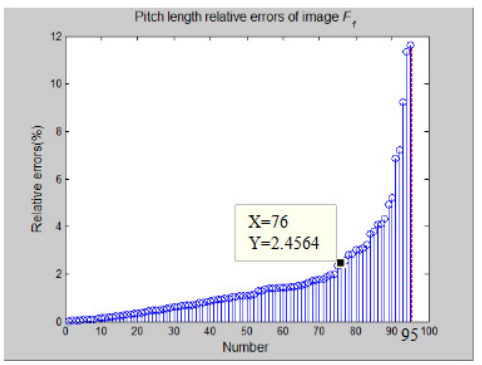

(c) Relative errors of measuring pitch lengths of $\mathrm{F}_{1}$

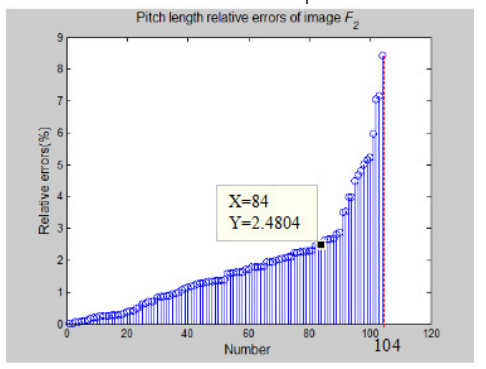

(f) Relative errors of measuring pitch lengths of $F_{2}$

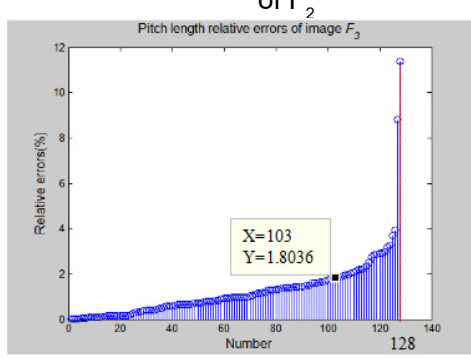

(i) Relative errors of measuring pitch lengths of $\mathrm{F}_{3}$

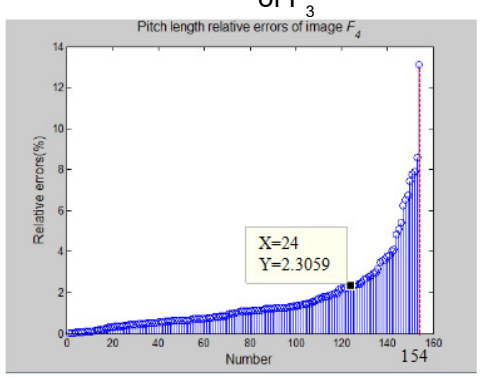

(I) Relative errors of measuring pitch lengths of $\mathrm{F}_{4}$

Figure 13. Corner detection results and relative errors of measuring pitch lengths and surface braiding angles

Table 2. Standard deviation of pitch length and surface braiding angle measured by hands

\begin{tabular}{|c|c|c|c|c|c|c|}
\hline & \multicolumn{2}{|c|}{ Standard deviation of pitch length } & \multicolumn{3}{c|}{ Standard deviation of surface braiding angle } \\
\hline & Minimum & Maximum & Average & Minimum & Maximum & Average \\
\hline$F_{1}$ & 0.026 & 0.10 & 0.061 & 0.40 & 0.90 & 0.64 \\
\hline$F_{2}$ & 0 & 0.056 & 0.022 & 0.27 & 0.88 & 0.53 \\
\hline$F_{3}$ & 0.018 & 0.063 & 0.040 & 0.34 & 1.15 & 0.73 \\
\hline$F_{4}$ & 0.018 & 0.062 & 0.036 & 0.31 & 1.065 & 0.62 \\
\hline
\end{tabular}

Table 3. Average values and relative errors of pitch length and surface braiding angle measurements

\begin{tabular}{|c|c|c|c|c|c|c|}
\hline & $\boldsymbol{d}_{\mathrm{ah}}(\mathbf{m m})$ & $\boldsymbol{d}_{\mathrm{aa}}(\mathbf{m m})$ & $\boldsymbol{\theta}_{\mathrm{ah}}\left({ }^{\circ}\right)$ & $\boldsymbol{\theta}_{\mathrm{aa}}\left({ }^{\circ}\right)$ & $\boldsymbol{e}_{\mathrm{da}}(\%)$ & $\boldsymbol{e}_{\theta \mathrm{a}}(\%)$ \\
\hline$F_{1}$ & 4.07 & 4.06 & 34.35 & 32.93 & 0.25 & 0 \\
\hline$F_{2}$ & 1.92 & 1.92 & 34.26 & 33.53 & 0 & 2.13 \\
\hline$F_{3}$ & 2.58 & 2.57 & 40.59 & 41.57 & 0.39 & 2.41 \\
\hline$F_{4}$ & 2.19 & 2.18 & 35.04 & 35.36 & 0.46 & 0.91 \\
\hline
\end{tabular}


regular braided composite preforms with small relative errors.

- The relative errors of pitch lengths measured by our method are smaller than that of surface braiding angles. The reasons are as follows. Firstly, the fabric crimp can affect the line angle determined by the two corners so as to affect the direction of the fiber bundle represented by the line; secondly, compared to the lengths, the angles are more sensitive to the changes of corner positions.

\section{CONCLUSIONS}

In summary, we achieved automatic measurement of pitch lengths and surface braiding angles of biaxial regular braided composite preforms based on the phase congruency. Some conclusions are drawn:

- The proposed method is not only suitable to measure pitch lengths and surface braiding angles of carbon-fiber fabrics, but also has good robustness to be applied to the measurements of color-fiber fabrics, such as glass- and aramid-fiber fabrics.

- The phase congruency covariance used in corner detection robustly pointed out the approximate locations of corners, which provides a good foundation for the subsequent corner detection.

\section{ACKNOWLEDGEMENTS}

The authors wish to thank Dr. Izabela Luiza Ciesielska-Wrobel for handling the review of the paper and the two anonymous reviewers for their helpful comments. The authors would like to thank Ms. Wei Li for fiber measurement device support.

\section{Funding}

This work was supported by Applied Basic Programs of China National Textile and Apparel Council (J201509).

\section{References}

[1] Qu, P., Guan, X., Jia, Y., et al. (2012). Effective elastic properties and stress distribution of $2 D$ biaxial nonorthogonally braided composites. Journal of Composite Materials, 46(8), 997-1008.

[2] Böhm, R., Hornig, A., Luft, J. et al. (2014). Experimental investigation of the strain rate dependent behaviour of $2 D$ biaxially and triaxially reinforced braided composites. Applied Composite Materials, 21(2), 285-299.

[3] Ayranci, C., and Carey, J. (2008). 2D braided composites: a review for stiffness critical applications. Composite Structures, 85(1), 43-58.
[4] Swanek, D. S. S., and Carey, J. (2007). Braided composite materials for the production of lightweight, high rigidity golf shafts. Sports Engineering, 10(4), 195-208.

[5] Du, G., and Popper, P. (1994). Analysis of a circular braiding process for complex shapes. Journal of the Textile Institute, 85(3), 316-337.

[6] Potluri, P., Manan, A., Francke, M., et al. (2006). Flexural and torsional behaviour of biaxial and triaxial braided composite structures. Composite Structures, 75(1), 377386.

[7] Xu, L., Kim, S. J., Ong, C. H., et al. (2012). Prediction of material properties of biaxial and triaxial braided textile composites. Journal of Composite Materials, 46(18), 22552270.

[8] Shokrieh, M. M., and Mazloomi, M. S. (2012). A new analytical model for calculation of stiffness of threedimensional four-directional braided composites. Composite Structures, 94(3), 1005-1015.

[9] Kostar, T. D., and Chou, T. W. (1994). Microstructural design of advanced multi-step three-dimensional braided preforms. Journal of composite materials, 28(13), 11801201.

[10] Goyal, D. and Whitcomb, J. D. (2006). Analysis of stress concentrations in $2 \times 2$ braided composites. Journal of composite materials, 40(6), 533-546.

[11] Wan, Z., Li, J. (2006). Braided angle measurement technique for three-dimensional braided composite material preform using mathematical morphology and image texture. AUTEX Research Journal, 6(1), 30-39.

[12] Gong, L., and Wan, Z. (2006). Automatic measurement technology on braided pitch length of three-dimensional braided composite material preform. Computer Measurement \& Control (in Chinese), 14(6), 730-733.

[13] Wan, Z., Shen, J., and Wang, X. (2004). Measure and research on braided angle of composites preform. Journal of Textile Research (in Chinese), 25(3), 42-44.

[14] Hernández-López, J. J., Quintanilla-Olvera, A. L., LópezRamírez, J. L., et al. (2012). Detecting objects using color and depth segmentation with kinect sensor. Procedia Technology, 3(1), 196-204.

[15] Dabov, K., Foi, A., Katkovnik, V., et al. (2006). Image denoising with block-matching and $3 D$ filtering. In: Proceedings of SPIE, Image Processing: Algorithms and Systems, Neural Networks, and Machine Learning, 6064, 1-12.

[16] Kovesi, P. (1999). Image features from phase congruency. Videre: Journal of computer vision research, 1(3), 1-26.

[17] Kovesi, P. (2003). Phase congruency detects corners and edges. In the Australian Pattern Recognition Society Conference: DICTA. Sydney, 309-318.

[18] Orfanidis, J. S. (1988). Optimum Signal Processing: An Introduction. (2nd ed.). Macmillan Publishing Company (New York). 